MICHAEL TYE

\title{
THE PROBLEM OF COMMON SENSIBLES
}

\begin{abstract}
Our experience of the qualities Locke classified as secondary qualities generates a problem, a version of which Aristotle raised. I call this problem "the problem of common sensibles." The problem, as I discuss it, concerns cross-modal experienced togetherness or unity. On the view that we undergo distinct sense-specific experiences as we hear, smell, taste, see, and touch things, there seems no room for cross-modal unity at the experiential level. But cross-modal unity is real and it necessitates that we give up the usual separatist view of sense experiences.
\end{abstract}

In On The Soul (425a-b), Aristotle drew a distinction between those qualities that are perceptible only via a single sense and those that are perceptible by more than one. The latter qualities he called "common sensibles". To lllustrate: suppose I am holding a banana. The shape of the banana is perceptible by my sight and also by my touch; but the color of the banana is perceptible only via sight. The shape is thus a common sensible; the color not.

Presumably, Aristotle was willing to allow that qualities that are not common sensibles are nonetheless sometimes detectable by more than one sense, as, for example, when I first infer a sweet taste in a red apple apprehended by sight and then experience that taste by biting into the apple. Aristotle's point was that some qualities are not themselves directly (that is, non-inferentially) given in experience to multiple senses, qualities like the redness of an apple and the sweetness of its taste, whereas others are so given.

The phenomenon of synthaesthesia may seem to create trouble for Aristotle's position here. PET scans on synaesthetes who are listening to words show increased blood flow not only in the auditory regions of their brains but also in brain areas dedicated to visual perception. The latter is not found in the brains of control groups listening to the same words (Harrison, 2001). For synaesthetes, it seems, colors are experienced not only by sight but also by hearing. But synaesthesia, of course, is an abnormality; and it could reasonably be responded that Aristotle's point is to be taken to apply only to normal perceivers. 
The distinction Aristotle drew between qualities that are common sensibles and qualities that are not is similar to one drawn later by John Locke between primary qualities - qualities with an objective, perceiver-independent nature - and secondary qualities - qualities whose essences are powers to generate, in normal perceivers, characteristic sense-specific phenomenological responses. Indeed, one possible explanation as to why colors, for example, are not common sensibles is that they are secondary qualities.

This is not the explanation I myself would offer; for I doubt whether any of the qualities to which the human senses are 'tuned' are secondary qualities, as understood by Locke. But this does not matter for my present purposes. Our experience of the qualities Locke classified as secondary qualities generates a problem, a version of which Aristotle himself raised. I shall call this problem the "problem of common sensibles."

\section{THE PROBLEM OUTLINED}

Here is one way of illustrating the problem. Let us return to the cases of the apple and the banana. The apple looks red and the banana looks yellow. The apple and the banana thereby look different colors. This difference in their color is given in visual experience. The apple not only looks red but also tastes sweet. The apple is thereby experienced as having two different qualities. The difference between redness and sweetness is as much given in experience as the difference between redness and yellowness. But how is this possible? Although there is a visual experience of redness and a gustatory experience of sweetness, there is no visual experience of both redness and sweetness and neither is there a gustatory experience of both these qualities. How, then, can there be an experience of their difference?

This way of putting the problem is the way Aristotle framed it. Unfortunately, it is not obviously correct to suppose, as Aristotle does, that the difference between sensible qualities, even within a single sense, is directly presented in experience. Suppose I see both the apple and the banana at the same time. I am aware of - I experience - the redness of the apple; likewise for the yellowness of the banana. But am I aware of - do I experience - the difference in color between the two? The expression 'the difference in color' is an abstract noun and abstract nouns following perceptual verbs usually (though not always) stand in for factive clauses. For example, in normal contexts to count as seeing the source of the trouble, it does 
not suffice that I see the thing that is in fact the source of the trouble (a malfunctioning thermostat). I must see that it is the source of the trouble. Similarly, to be aware of the difference in color of the apple and the banana, I must be aware that the apple and the banana differ in color. Arguably, my awareness 'of' the difference in color is merely a judgement that the apple and banana differ in color on the basis of my experience of their colors. This is not to say that the judgement is an inference. The basing relation that connects the judgement to the color experiences is evidential but it need not be inferential. The point is rather that the difference in color is not itself directly experienced. And if this is so, then the problem, as presented above, is based on a false assumption.

However, the problem need not be formulated in the way that Aristotle himself did. Instead of focusing on experienced difference across senses, we can focus on what we might call "experienced togetherness".

Suppose that I hear a loud noise and I simultaneously see a yellow flash. It is not an accurate account of the first person perspective to say simply that I have an auditory experience of something loud and simultaneously I have a visual experience something yellow. For it is part of the phenomenology of my experience that the loudness of the noise and the yellowness of the flash are experienced together (assuming that the case is a normal one). Their togetherness is as directly and immediately given to me in my experience as the togetherness of the redness and roundness of the apple's surface. In the latter case, it does not do justice to the character of my experience to say merely that I have an experience of a red surface and also an experience of a round surface. Similarly, in the former. How can this be? If loudness is experientially 'trapped' in one sense and yellowness in another, how can the two be experienced together?

One might reply that what I am calling "experienced togetherness" or unity is simply a matter of the relevant experiences being directed upon the qualities of a single object in a spatially localized region. This certainly fits with the case of the redness and roundness of the apple; but it does not accommodate the case of the noise and the flash. Consider also the following case involving experiences directed upon widely separated objects. Standing by the railing of a ship and smelling the sea air, as I look at the ship's wake in the ocean, I hear the sound of a tugboat from afar. Intuitively, it is not simply that I have an experience of a vivid blue color and also an experience of a salty smell and further an experience of a booming sound. Color, smell, and sound are experienced together; there is, as it were, 
a seamless phenomenal whole within which these qualities are phenomenologically present. Again: how can this be?

A reaction some philosophers may have to these examples is to say that they should be treated in essentially the same way as the case of experienced difference. On this view, the senses deliver up sensespecific experiences which then get operated on by the cognitive faculty. Thought is really what joins the sense-specific experiences. This neo-Kantian view seems to me implausible, however. Experience itself is not phenomenologically fragmented in the way this view requires. Intuitively, the unity or togetherness is presented in experience, whatever its subjects can or cannot think. It is certainly true, of course, that without the relevant concepts, a person cannot recognize that there is both a loud noise and a yellow flash, for example, and thus, in these circumstances, he or she is "blind" to the unity in her experience. But the unity itself is not created by the act of recognition any more than is the experience.

It should be clear from my remarks thus far that the relation of experienced togetherness, as I am understanding it, is a phenomenal relation. There is something it is like to experience the smell of the sea air, for example; and there is something it is like to experience the color of the sea; but there is also something it is like to experience these things together. Moreover, in saying that the relation of experienced togetherness (or unity or co-presence in consciousness) is phenomenal, I mean to distinguish it conceptually from spatial unity (as described in the apple case), neurophysiogical unity (which obtains just in case the relevant experiences are realized by a single neurophysiological mechanism), subject unity (the relata of which are experienced by the same subject), higher-order subject unity (the relata of which the subject can self-ascribe as being experienced at a given time), introspective unity and general attentional unity.

What, then, is the solution to the problem of common sensibles? In Section 2, I shall propose an answer. Section 3 responds to objections. The final section sketches a view of the phenomenal character of experience and phenomenal unity which goes naturally with the proposal made in Section 2.

\section{THE PROBLEM SOLVED}

The problem of common sensibles is generated initially by an assumption widely shared in both philosophy and psychology that the senses function as largely separate channels of information which 
generate different sense-specific impressions or experiences. For example, I see some flowers, a fence, two squirrels, and in seeing them, I undergo visual experiences. Listening to a nearby bird singing, I hear melodious sounds and in so doing, I am the subject of auditory experiences. Finding a broken egg on the ground, and smelling its pungent odor, I experience olfactory experiences. Placing a chocolate in my mouth and tasting its sweetness, I have gustatory experiences. Running my fingers over the bark of a tree and feeling its roughness, I experience tactual experiences.

According to this view, if I am using all five of my senses at a given time, I undergo five different simultaneous perceptual experiences at that time, each with its own distinctive sense-specific phenomenal character. Since there is in this picture as yet no place for experiences that encompass multiple secondary qualities (or so-called "secondary qualities") associated with different senses, we may now naturally be puzzled as to how we can experience such qualities together.

Aristotle's own solution was to say that the picture is incomplete: in addition to the sense-specific faculties, there is a faculty of common sense. Using this faculty, perceivers are able to make cross-modal comparisons between qualities such as redness and sweetness. Exactly, what Aristotle had in mind here is not clear; but his thought may well have been that in addition to the sense-specific experiences generated by the use of the five senses, there is a further overarching experience of qualities that are not common sensibles generated by the faculty of common sense. This experience forms the basis for cross-modal comparisons between such qualities.

Note that the overarching experience cannot just be a conjunction of the five modality-specific experiences. The conjunction of two experiences isn't itself an experience at all. The overarching experience must be a new experience, one that unifies the qualities experienced via different senses into a single phenomenological whole.

One obvious question that faces the above proposal concerns the nature of the overarching experience in relation to the other experiences. ${ }^{1}$ There is also a further question as to whether, once the move has been made to introduce a common experience, there remains any need to countenance the sense-specific experiences too. Let me explain.

Consider the following example (from Parsons, 1972). Suppose that this statement is true:

(S) Jones writes illegibly and Jones writes painstakingly.

It does not follow that

(S*) Jones writes illegibly and painstakingly 
is true, at least on one natural reading of $\left(\mathrm{S}^{*}\right)$. For if Jones writes illegibly but not painstakingly with his left hand and painstakingly but legibly with his right, then $(\mathrm{S})$ is true but $\left(\mathrm{S}^{*}\right)$ false. In this case, there is an event of Jones' writing illegibly and there is an event of Jones' writing painstakingly, but these are two distinct events. There is no event of Jones' writing both illegibly and painstakingly. So, (S) does not entail $\left(\mathrm{S}^{*}\right)$, but $\left(\mathrm{S}^{*}\right)$ clearly does entail (S). Given the event of Jones' writing both illegibly and painstakingly, there is, of course, the event of Jones' writing illegibly. For the latter is the very same event as the former under a less broad description. Likewise for the event of Jones' writing painstakingly.

In the case where $\left(\mathrm{S}^{*}\right)$ is true, there is a kind of unity to Jones' writing. Illegibility and painstakingness are combined together in a single instance of writing. That unity is lacking in the case that $(\mathrm{S})$ is true and $\left(\mathrm{S}^{*}\right)$ is false. But where $\left(\mathrm{S}^{*}\right)$ is true, there aren't two different writings, one painstaking and the other illegible, which somehow are unified together to produce a third, overarching writing that includes them. There is just one writing that may be described in more or less encompassing ways. ${ }^{2}$

Here is another example. Suppose it is lunchtime and I have a sudden and strong desire for a pint of beer with a ham sandwich. In having this desire, of course, I have a desire for a pint of beer. It is also true that I have a desire for a ham sandwich. But patently I don't have three sudden desires here. Nor is it the case that having a desire for a beer together with a desire for a ham sandwich just is having a desire for a pint of beer with a ham sandwich. I might want a beer and also want a ham sandwich while finding the idea of having the two together repellent.

My sudden desire for a pint of beer with a ham sandwich is a single desire that can be described in multiple ways. The description "desire for a pint of beer" is incomplete, but unlike the description "desire for a pint of beer alone", it is not inaccurate.

These remarks apply mutatis mutandis, I want now to suggest, to the problem of common sensibles. There aren't five different or separate simultaneous experiences somehow combined together to generate a further unified experience. To be sure, if I am witnessing a yellow flash and a loud noise, the statement

(S!) I have an experience of a yellow flash and I have an experience of a loud noise is true. And, given that the case is a normal one, the following is true too:

(S\#) I have an experience of a yellow flash and a loud noise. 
Moreover, (S\#) entails (S!). But there is just one experience here, an experience that can be described less fully as my experience of a yellow flash or as my experience of a loud noise.

On this view, there really are no such entities as purely visual experiences or purely auditory experiences or purely olfactory experiences, etc in normal, everyday consciousness. Where there is experienced togetherness across sense modalities, sense-specific experiences do not exist. They are the figments of philosophers' and psychologists' imaginations. And there is no problem, thus, of connecting these experiences up with an overarching experience. There are no sensespecific experiences to be connected. There is just a single multi-modal experience, describable in more or less rich ways. The problem of common sensibles now dissolves.

\section{OBJECTIONS TO THE ONE EXPERIENCE VIEW}

"Stuff and nonsense", you may say. "The proposal is empirically false. Visual experiences are known to arise in the visual cortex, auditory experiences are known to arise in the auditory cortex, and so on. In the case of a winetaster, say, who sees and smells and tastes a wine, it is surely undeniable that visual experiences are tokened in the winetaster's visual cortex, as he views the wine he is tasting, experiences of just the same phenomenal type as those that would have been tokened in that cortex, had the situation been the same but his other senses blocked from any information. Of course, these experiences exist!"

By way of reply, let us for a moment indulge in the fiction that there are purely visual experiences in everyday consciousness of the external world. In these experiences, shape and color are unified. If, for example, I view a green square, my visual system represents the greenness and the squareness in separate places in the brain; but these qualities aren't experienced as separate. They are experienced as qualities of a single thing. I have an experience that is object-unified, as we might say, even though its physical basis is disunified. ${ }^{3}$ What is the relationship between the experience and its physical basis? The answer I favor is that the experience, assuming there is one, is constituted by a certain combination of separate and largely independent physical events in the visual cortex, but it is not token identical with that combination. This answer gives the experience a physical nature; moreover constitution is the relation that bonds macro-events and 
micro-events, macro-states and micro-states, macro-objects and micro-objects generally.

Consider, for example, a single cloud in the sky. The cloud is an aggregate of water droplets. The "is" in the last sentence is not the "is" of identity. The cloud in the sky could survive the loss of a few of its constituent water droplets (if, say, a highly localized strong gust of warm air were to cause them to evaporate). Not so any aggregate of water droplets that contains them. The loss of those droplets would destroy the original aggregate. So, the cloud has a modal property the aggregate of water droplets lacks, that of possibly surviving the loss of such-and-such droplets. It follows by Leibniz' Law that the cloud is not identical with any aggregate of droplets. In general, ordinary, everyday macro-objects are not identical with aggregates of their parts, since the former differ in their modal properties from the latter. My car, for example, might have had a different carburettor, but the aggregate of its actual parts could not have failed to contain the actual carburettor. The car, thus, is not identical with the sum of its parts. The relationship rather is one of constitution or composition.

One need not resort to modal properties to make the above points. Actual properties will do in some cases. The clay that constitutes a pot exists before the pot does. The lump of silver that is melted and formed into a coin exists before the melting process, but the coin does not. The clay is thus not identical with the pot; the lump of silver not identical with the coin.

Likewise for macro-events. Consider the eruption of Mount Vesuvius. Intuitively, that very eruption might have spewed forth an imperceptibly smaller amount of lava. Had it done so, the micro-events taking place in the spatial region of Vesuvius' eruption would have been minimally different and thus the aggregate of those events in that counterfactual situation is not the same as the actual aggregate. The eruption, therefore, has a modal property that the underlying cluster of micro-events lacks, and the former is not identical with the latter.

Alternatively, consider all the micro-physical events that compose the emergence of North America (Burge, 1986). Imagine that these events are embedded within a much larger land mass, so that in the counterfactual situation there is no such thing as North America and its emergence. Then the aggregate of micro-events has the property of possibly existing without North America; but the event of North America's emerging does not. That event is not identical with the aggregate. The aggregate constitutes the emergence of North America in actual fact, but the relationship is not one of identity. 
Consider next the following example. A large chunk of clay is used to make a statue at time $t$. The clay constitutes the statue without being identical with it. Suppose counterfactually that at time $t$ ', where $t^{\prime}$ is later than $t$, an artist cleverly removes much of the clay without remolding it so as to leave behind a small clay pot. In the counterfactual situation, the clay that remains constitutes a pot at $t^{\prime}$. But in the actual situation it does not. In actual fact, no clay is removed. There is, in actual fact, no tiny pot within the statue. There is only the statue.

Within the aggregate of lumps of clay composing the statue, there is a smaller aggregate of clay lumps that in a certain counterfactual situation composes a pot. In actual fact, the smaller aggregate does not compose a pot. Indeed, it does not by itself actually compose or constitute any ordinary thing. Rather that aggregate together with the remaining aggregate form a larger aggregate that composes the statue.

I hope that the relevance of all of this is becoming clear. On my view, at the given time the winetaster mentioned above is subject to a single experience that represents the color of the wine, the smell of the wine, the taste of the wine, etc. This experience is constituted by a combination of largely independent physical events going on in separate regions of the brain. Within that combination of events, there is a cluster of events (call it " $C$ ") occurring in the winetaster's visual cortex. In the extraordinary counterfactual situation in which the winetaster's nonvisual senses are all blocked, so that no nonvisual information gets in, the winetaster is left with a purely visual experience. And in that counterfactual situation, $C$, in the absence of the other pertinent actual physical events, constitutes a visual experience. But it does not follow from this that in actual fact $\mathrm{C}$ constitutes a purely visual experience. In actual fact, $C$ (wholly) constitutes no experience at all. There is just one experience the winetaster undergoes, and $C$, in conjunction with the relevant events in other parts of the brain, e.g., the olfactory cortex, etc, constitutes that. $^{4}$

Perhaps it will be replied that in the example of the statue and the pot, the aggregate of lumps of clay that counterfactually constitutes the pot is in the actual world a purely arbitrary part of the statue, with nothing to mark it out from any number of other arbitrary parts of the statue. However, the cluster of physical events I have labeled " $C$ ", is a nonarbitrary part of the relevant totality of physical events, a token of a physical type with a definite functional role, namely to generate a conscious visual experience with a certain visual unity. 
This begs the question. I grant that $C$ is a token of a physical type $P$ whose role in a normally functioning brain is to endow the conscious experience of the subject with a visual phenomenology. But that is certainly compatible with denying that $P$ 's role is to generate in the brain a token experience with an exclusively visual phenomenology. To suppose that $P$ 's role is the latter is to take for granted the truth of the view I am opposing.

Furthermore, the fact that $C$ is a nonarbitrary part of the whole combination of physical events constituting the experience is not to the point. Suppose events $E 1$ and E2 together actually constitute event $F$. Suppose $E 1$ could have occurred without $E 2$ and further that had $E 1$ done so, it would have (wholly) constituted event $G$. Still, this is no guarantee that $E 1$ actually constitutes G. For example, my arm and hand movement relative to Smith and Smith's arm and hand movement relative to me constitute a certain fight. Smith's movement might have occurred without my movement. Had it done so, it would have constituted an act of aggression on the part of Smith. But in actual fact that act of aggression does not exist. In actual fact, Smith's arm and hand-movement relative to me is a counterpunch; for I hit Smith first.

Thus, just as Smith's arm and hand movement might have constituted an act of aggression although in reality it does not, so too the cluster of events, $C$, might have constituted a purely visual experience but in reality it does not.

Suppose now that the counterfactual situation I have envisaged for $C$ becomes a reality. As the winetaster tastes the wine, some extraordinary neural malfunction causes the events other than $C$ that constituted the winetaster's experience prior to the malfunction to cease. Before the malfunction, on the proposed view, $C$ does not constitute a purely visual experience. After the malfunction, it does. This, it may be charged, is strange. Why the radical change in what $C$ does?

The answer, as earlier, is that there is no change. But before and after the malfunction, $C$ does the same thing: it endows the conscious experience of the subject with a visual phenomenology. The difference is that before the malfunction, the experience of the winetaster does not have a purely visual phenomenology; after the malfunction, it does.

Consider next the following example. Suppose I hear a conversation on my left, as I look at a bed of roses laid out in front of me. Intuitively, my auditory experience $C$ that very experience $C$ could have occurred without my visual experience. On my account, however, that isn't possible. So much the worse, it may be said, for my account! 
Too fast, I reply. When I try to attend to my auditory experience via introspection, what I actually come across are the sounds and the auditory qualities the experience represents. By being aware of those sounds, I am aware that I am undergoing an auditory experience. But I am no more aware of the token vehicle of that content than I am of a burglary when, upon returning home, I see signs of a forced entry on the front door of my house. My experience thus is transparent to me (Dretske, 1995; Tye, 1995, 2000).

If this is correct, then it is not intuitively clear that my auditory experience could have occurred without my visual experience. The sounds I experience could have existed without my visual experience of the roses. Moreover, I certainly could have undergone an experience that represented those sounds (or sounds just like them) without also representing the colors and shapes of roses. But it is, of course, perfectly compatible with these claims that I am the subject of just one experience, an experience that is audio-visual in character.

What if my auditory experience goes on longer than my visual experience? Then, it may be urged, my auditory experience has a temporal property my visual experience lacks, and there cannot be a single experience after all.

I grant that I can experience a sound that continues, in my experience, after I experience anything visually. But this is all in the content of the experience. Initially, what I experience is that a sound with a certain pitch and loudness is accompanied by a certain color and shape. As time goes on, the experienced content changes. No longer is any shape or color represented. The sound is represented on its own. This certainly shows that the represented sound is not the same as the represented shape or color. But it does not show that there is more than a single experience at a time.

I do not deny, of course, that difficult questions arise concerning the individuation of experiences through time. Is the experience I undergo initially - an experience with an audio-visual content - the same as the experience I undergo after the color and shape cease? Is there one experience here with a less rich content through time? Or is the audio-visual experience replaced by a second purely auditory experience, phenomenally just like the first in its auditory dimension? Hard questions of individuation through time arise for everyone, however. I shall not discuss them here.

Let me quickly mention one final worry. Seeing something entails the presence of a visual experience. I cannot see $X$ unless $X$ looks some way to me; and for $X$ to look some way to me, it must cause in me a visual experience. However, on the account I am adopting, in 
normal cases, when I see something, my experience isn't really properly classified as visual at all.

It is indeed true that $X$ cannot look some way to person $P$ unless $X$ produces in $P$ an experience with a visual phenomenology. But the phenomenology of $P$ 's experience need not be purely or exclusively visual. It can be partly auditory, olfactory, gustatory, and tactual too. If a visual experience is understood to be an experience with a visual phenomenology, then, as I see something, I am subject to a visual experience. It's just that that very experience has a phenomenology that is auditory, olfactory, gustatory, and tactual as well.

4. FURTHER ISSUES: PHENOMENAL CHARACTER AND PHENOMENAL UNITY

Those who think of the phenomenal character of an experience what it is like to undergo the experience - as a quality of the experience think of phenomenal unity or togetherness as a relation between experiences, a binding relation that unifies sense-specific experiences into an overarching maximal experience. This is not how I think of phenomenal unity. The core intuition, lost in the usual way of understanding phenomenal unity and emphasized earlier in this essay, is that, in normal cases, simultaneously experienced perceptual qualities $\mathrm{C}$ the loudness of a sound, the smoothness of a surface, the sweetness of a taste, the pungency of a smell $\mathrm{C}$ are experienced together and thus are phenomenologically unified. These qualities are not qualities of experiences. They are qualities that, if they are qualities of anything, are qualities of things experienced. Phenomenal unity or togetherness is a relation between qualities represented in experience, not between qualities of experiences.

Where phenomenal unity obtains, perceptual experience is closed under conjunction with respect to the unified qualities. Thus, in the case in which the loudness of a sound is phenomenally unified for person $P$ with the brightness of a flash of light, the statements

$P$ has an experience of a loud sound

and

$P$ has an experience of a bright flash

jointly entail

$P$ has an experience of a loud sound and a bright flash. 
When there is disunity, perceptual experience is not closed in this way. Cases of simultaneous phenomenal disunification all involve multiple perceptual experiences at a time and multiple experiential contents. Such cases are highly abnormal. Where they occur, as, for example, with split brain patients, there are simultaneously experienced perceptual qualities entering into different contents (each of which is a content experientially represented by the relevant subject at the given time). ${ }^{5}$

Here is not the place for a full account of phenomenal unity; but very briefly my view is that phenomenal unity is a matter of the simultaneously experienced perceptual qualities entering into the same phenomenal content. The perceptual experience a normal perceiver undergoes has an enormously rich, multi-modal representational content - a content which, insofar as it is distinctively experiential, is nonconceptual, abstract, and appropriately poised. ${ }^{6}$ This content is the phenomenal content of the experience. It is present not only in veridical cases but also in cases of illusion and hallucination. It is this content that endows the experience with its phenomenal character (Tye, 1995, 2000).

Interestingly, the case of split brain patients provides the basis for an argument that the relation of phenomenal unity is non-transitive, and this in turn lends further support to the account I am suggesting of phenomenal unity. I want now in closing to elaborate upon this point.

The operation of cutting the corpus callosum (a large strand of neurons connecting the left and right hemispheres of the brain) was originally performed by Roger Sperry in the 1960s on some epileptic patients, with the aim of controlling epileptic seizures. It had a remarkable consequence. In addition to reducing greatly the number and intensity of the seizures themselves, it also produced a kind of mental bifurcation in the epileptic patients.

Here is an illustration. A subject, $S$, is told to stare fixedly at the center of a translucent screen which fills his visual field. Two words are flashed onto the screen by means of a projector located behind, one to the left of the fixation point and one to the right, e.g., the words "pen" and "knife". The words are flashed very quickly (for just $1 / 10$ of second) so that eye movements from one word to the other are not possible. This arrangement is one that ensures that the word on the left provides input only to the right hemisphere of the brain and the word on the right provides input only to the left.

$\mathrm{S}$ is then asked what he saw. $\mathrm{S}$ shows no awareness, in his verbal responses, of "pen". However, if S is asked to retrieve the object 
corresponding to the word he saw from a group of objects concealed from sight, using his left hand alone, he will pick out a pen while rejecting knives. Alternatively, if $\mathrm{S}$ is asked to point with his left hand to the object corresponding to the word he saw, he will point to a pen. Moreover, if $\mathrm{S}$ is asked to sort through the group of objects using both hands, he will pick out a pen with his left and a knife with his right. In this case, the two hands work independently with the left rejecting the knives in the group and the right rejecting the pens.

Once the corpus callosum is cut, the two hemispheres are not wholly divorced from one another; for the sub-cortical pathways still link the two. For example, if a pornographic picture is presented only to the right hemisphere, the subject reports feeling differently. "That's quite a machine you've got there," one split brain patient remarked. Since verbal responses are controlled by the left hemisphere, information about the picture is evidently getting there. In another experiment, the subject saw, with his right hemisphere only, a picture of a frightening scene of a fire. Afterwards, he commented, "I don't really know what I saw; I think just a white flash. Maybe some trees, red trees like in the Fall. I don't know why, but I feel kind of scared. I feel jumpy. I don't like this room, or maybe it's you guys getting me nervous." Again, what seems to have happened is that the emotion triggered by the right hemisphere had an effect, via the brainstem, on the verbal left hemisphere.

The sub-cortical pathways in the brainstem also are responsible for the experience of touch around the neck and on the head. For example, if a split brain patient is asked to say where a pin is gently pricking him on his neck or face, he will report its location accurately. If asked to touch his left forefinger to the relevant spot after the pin has been removed, again he will do so accurately. Here, the split brain patient's behavior is just the same as that of a normal subject. And just as the verbal and nonverbal reponses of the normal subject are evidence for the experience of a pin prick in a certain location, so too are those of the split brain subject.

Imagine now that as the neck of the split brain subject, $\mathrm{S}$, is pricked, he is presented with a red screen to the left, which he touches with his left forefinger, and a green screen on the right, which he touches with his right forefinger. $\mathrm{S}$ has an experience of his left forefinger together with a red surface. He also experiences that forefinger as connected to his wrist and his arm; and he experiences his arm as connected to his neck, in which he experiences the prick. The same is true for $\mathrm{S}$ 's right forefinger, except that now the relevant surface is the green one. $S$, thus, experiences the redness of the surface 
and the pricking of the pin together. They are phenomenally unified in his experience. Likewise, $\mathrm{S}$ experiences the greenness of the other surface and the pricking of the pin together. They also are phenomenally unified in his experience. But redness and greenness are not experienced together. They are not phenomenally unified. Accordingly, phenomenal unity is not transitive: there are cases in which $A$ is unified with $B, B$ is unified with $C$, but $A$ is not unified with $C$. How is this to be accounted for?

Within the general framework elaborated above, the explanation is straightforward. $\mathrm{S}$ has two multimodal experiences, $E_{1}$ and $E_{2} . E_{1}$ represents the pinprick in S's neck, his left arm, fingers and the surface his left forefinger is touching. $E_{2}$ represents the pinprick in S's neck, his right arm, fingers and the surface the right forefinger is touching. The pricking is phenomenally unified with redness by their entering into the same phenomenal content $C$ the phenomenal content of $E_{l}$. The pricking is phenomenally unified with greenness in like manner, but this time the common content is the phenomenal content of $E_{2}$. Since $\mathrm{S}$ has no experience whose phenomenal content has entering into it both greenness and redness, the two colors are not phenomenally unified.

There is thus no more mystery attaching to the non-transitivity of phenomenal unity than there is to the non-transitivity of the relation of entering into the same thought content. Just as I can consciously think that Ann loves Paul and also consciously think that Paul is a neighbor of Raoul without having any conscious thought into whose content Ann and Raoul enter, so I can experience $A$ unified with $B$ and also experience $B$ unified with $C$ without my experiencing $A$ and $C$ together.

It is not easy to see how the non-transitivity of the phenomenal unity relation is to be explained on alternative accounts. Those who, in opposition to the view I have proposed, think of unity or togetherness as a relation between experiences will apparently have to say that non-transitivity is a primitive feature of the relation. But brute facts such as this are surely to be avoided wherever possible. Alternatively, it might be argued that the case just adumbrated does not suffice to show non-transitivity on the grounds that there are actually two simultaneous token experiences of the pin prick in the neck. One of these experiences is unified with the experience of a green surface and the other with the experience of a red one.

The biggest difficulty here, leaving aside the point that unity, or so I have claimed, is not a relation between experiences at all, is that there is no more reason to postulate two token, phenomenally identical experiences of the relevant region of the neck of the split brain subject than 
there is to make such a postulation for any subject. Just as the latter postulation for normal minds is unmotivated, unnecessarily complex, and perhaps even incoherent (depending on how token experiences are individuated), so too is the former. Much better to take the case presented at face value and give up what is, from the present perspective, a purely dogmatic adherence to transitivity.

\section{NOTES}

${ }^{1}$ For difficulties that arise here, see Tye (2003).

2 Not everyone accepts this claim. On the view of events elaborated by Jaegwon Kim (1976) and Alvin Goldman (1970), the event of $x$ 's $F$-ing $G$-ly is a complex entity consisting of $x$ and the property of $F$-ing $G$-ly. This has the consequence that at the time at which (S2) is true, there are four relevant simultaneous events: Jones' writing painstakingly and illegibly, Jones' writing painstakingly, Jones' writing illegibly and Jones' writing. Intuitively, however, events do not individuate in this hyper-finegrained way.

${ }^{3}$ I am not suggesting here that object-unity is the same as phenomenal unity. See earlier, Section 2.

${ }^{4}$ In my view, there is a maximality constraint on experiences just as there is on clouds and statues and pictures. Consider a visual experience of a scene $\mathrm{S}$. It is generally agreed that this does not contain many token visual experiences of parts of S. Why treat the multi-modal case any differently?

${ }^{5}$ For a discussion of split brains, see Tye (2003, ch. 5). In my view, it is a mistake to suppose that the perceptual consciousness of split brain subject is always divided after the commissurotomy. Indeed, it is a mistake to suppose that even in the highly specialized circumstances in which split brain subjects typically behave anomalously, their consciousness is invariably divided.

${ }^{6}$ For an elucidation of what it is for a content to be nonconceptual, abstract, and poised, see Tye $(1995,2000)$. For further critical discussion and replies, see the web symposium on Tye (2000) at http://host.uniroma3.it/progetti/kant/field/ tyesymp.htm.

\section{REFERENCES}

Aristotle: 1973, 'On the Soul', in R. McKeon (ed.), Introduction to Aristotle, University of Chicago Press, Chicago.

Burge, T.: 1986, 'Individualism and Psychology', Philosophical Review 95, 3-45.

Dretske, F.: 1995, Naturalizing the Mind, MIT Press, Cambridge/MA, Bradford Books.

Goldman, A.: 1970, A Theory of Human Action, Prentice-Hall, Englewood Cliffs.

Harrison, J.: 2001, Synaesthesia: the Strangest Thing, Oxford University Press, Oxford. 
Kim, J.: 1976, 'Events as Property Exemplifications', in M. Brand and D. Walton (eds.), Action Theory, Reidel, Dordrecht, pp. 159-177.

Parsons, T.: 1972, 'Some Problems Concerning the Logic of Grammatical Modifiers', in D. Davidson and G. Harman (eds.), Semantics of Natural Language, Reidel, Dordrecht, pp. 127-141.

Tye, M.: 1995, Ten Problems of Consciousness: A Representational Theory of the Phenomenal Mind, The MIT Press, Cambridge/MA, Bradford Books.

Tye, M.: 2000, Consciousness, Color, and Content, The MIT Press, Cambridge/MA, Bradford Books.

Tye, M.: 2003, Consciousness and Persons: Unity and Identity, The MIT Press, Cambridge/MA.

Department of Philosophy

University of Texas at Austin,

Austin, TX, 78750, USA

E-mail: mtye@mail.utexas.edu 